\title{
Programa Previne Brasil: o ápice das ameaças à Atenção Primária à Saúde?
}

\author{
Previne Brasil Program: the apex of threats to Primary Health Care?
}

Marismary Horsth De Seta (https://orcid.org/0000-0003-4764-5159) ${ }^{1}$

Carlos Octávio Ocké-Reis (https://orcid.org/0000-0003-4666-7575) ${ }^{2}$

André Luis Paes Ramos (https://orcid.org/0000-0003-3747-2207) ${ }^{3}$

${ }^{1}$ Escola Nacional de Saúde Pública, Fundação Oswaldo Cruz. R. Leopoldo Bulhões 1480, Manguinhos. 21041-210 Rio de Janeiro RJ Brasil.

deseta@ensp.fiocruz.br ${ }^{2}$ Diretoria de Estudos e Políticas Setoriais de Inovação e Infraestrutura, Instituto de Pesquisa Econômica Aplicada. Rio de Janeiro RJ Brasil.

${ }^{3}$ Coordenação Geral de Contratualização, Controle e Auditoria, Secretaria Municipal de Saúde do Rio de Janeiro. Rio de Janeiro RJ Brasil.

\begin{abstract}
Since 2017, the Brazilian health system is facing a wave of counter-reforms in policies that have expanded coverage and access and intended to change the care model. Primary Health Care (PHC) has been substantially modified by synergistic and complementary federal official acts. The creation of federal autonomous social service for the provision of doctors; public consultation to institute basket of consumption in healthcare; the flexibility of the weekly workload of doctors and nurses, who may integrate more than one team; the non-setting of a minimum number of community health workers per team form the context in which the Previne Brasil Program was launched to be in force by 2020. The government's argument is to increase: flexibility and local autonomy to organize services, greater efficiency and valorization. of performance. Criticism from sanitarians, some state councils of municipal authorities and the National Health Council points to the privatizing, marketing, selective and focused character of the proposal that is moving towards universal health coverage. This paper analyzes the Previne Brazil Program which, among other thin$g$ s, alters funding and suggests increasing resources for PHC in a context of freezing social spending. Contradictions and alternatives are identified to minimize potential damage to existing policies.
\end{abstract}

Key words Healthcare financing, Financial resources in health, Primary Health Care
Resumo Desde 2017, o sistema de saúde brasileiro enfrenta uma onda de contrarreformas em políticas que ampliaram cobertura e acesso e pretenderam mudar o modelo assistencial. A Atenção Primária à Saúde (APS) tem sido atingida por atos oficiais, sinérgicos e complementares, que a alteram substancialmente. A criação de serviço social autônomo federal para provisão de médicos; a consulta pública para instituir cesta de serviços; a flexibilização da carga horária semanal de médicos e enfermeiros, que poderão integrar mais de uma equipe; a não fixação de quantitativo mínimo de agentes comunitários de saúde por equipe; formam o contexto em que o Programa Previne Brasil foi lançado para vigorar em 2020. O argumento do governo é de aumento de flexibilidade e autonomia local para organizar serviços, maior eficiência e valorização do desempenho. Críticas de sanitaristas, de alguns conselhos estaduais de gestores municipais e do Conselho Nacional de Saúde apontam o caráter privatizante, mercadológico, seletivo e focalizado da proposta que caminha na direção da cobertura universal em saúde. Este artigo analisa o Programa Previne Brasil que altera o financiamento e sugere aumento de recursos para APS numa conjuntura de congelamento do gasto social. Identificam-se contradições e alternativas.

Palavras-chave Financiamento da assistência à saúde, Recursos financeiros em saúde, Atenção Primária à Saúde. 


\section{Introdução}

Após o golpe jurídico-midiático-parlamentar ${ }^{1}$ que destituiu Dilma Rousseff em 2016, houve clara inflexão na diretriz ideológica da condução das políticas sociais nos governos subsequentes, com novas medidas implantadas no governo $\mathrm{Te}$ mer e intensificadas após a eleição de Bolsonaro.

Apesar de a adoção de modelo de ajuste fiscal ter se dado ainda no período de Dilma Rousseff na tentativa de suplantar a crise política e econômica, após o golpe se concretizaram ações como a aprovação da Emenda Constitucional (EC) 95, que congela o teto dos gastos sociais, e as Reformas Trabalhista e Previdenciária.

Em 2017 e 2019, com foco na Atenção Primária à Saúde (APS), emitiram-se portarias ministeriais, consulta pública e Medida Provisória (MP), complementares e sinérgicas, que desidrataram princípios do SUS e minaram processos de planejamento, financiamento e provisão de ações e serviços.

A Política Nacional de Atenção Básica² ${ }^{2}$ passa a remunerar Equipes de Atenção Básica (eAB) formadas por médicos, enfermeiro, auxiliar ou técnico de enfermagem; faculta a presença de dentistas, auxiliares e técnicos de saúde bucal, agentes comunitários de saúde (ACS) e de controle de endemias (ACE); e fixa a carga horária mínima por categoria profissional na eAB em 10 (dez) horas e o máximo de três profissionais por categoria com 40 horas/semanais. Para as Equipes de Saúde da Família (eSF), ela deixa de estabelecer o mínimo de quatro ACS por equipe, que poderão aferir sinais vitais e glicemia e realizar curativos. Nova norma ${ }^{3}$ estabeleceu como componente mínimo da eAB, agora chamada Equipe de Atenção Primária (eAP), o médico e o enfermeiro, com 20 ou 30 horas semanais; permitir a sua participação em mais de uma eSB, eAP ou eSF, sem risco de suspensão de repasse; flexibilizar a carga horária das equipes de saúde bucal (eSB) nos mesmos moldes.

A transferência de recursos federais nos blocos de custeio e de investimento ${ }^{4}$, a despeito da prestação de contas se organizar nos seis antigos blocos, fragiliza a proteção do financiamento da APS frente à pressão dos custos da atenção hospitalar e especializada, e de procedimentos de diagnose e terapia, com forte componente privado ${ }^{5}$.

A Carteira de Serviços para a Atenção Primária à Saúde do Brasil ${ }^{6}$ objetiva "estabelecer um cenário 'ótimo' para a implementação e oferta de serviços clínicos nas unidades de saúde" e o "fortalecimento da clínica da APS com base científica para tomada de decisão". Ela prevê: a) rol de serviços de Atenção à Saúde (Adulto/ Idoso; Criança, Procedimentos na APS e Saúde Bucal); b) remuneração por capitação mediante "uma lista de pacientes" e "pagamento por pessoa acompanhada"; c) cesta mínima de serviços; d) detalhamento da organização do Programa "Saúde na Hora", em que as unidades básicas de saúde (UBS) funcionam 60 a 75 horas por semana; e) secundariza objetivos de promoção da saúde na APS, exemplificada mediante "o enfermeiro, que historicamente encontra-se focado na realização de atividades repetitivas e pouco eficazes centradas na promoção e prevenção de saúde em detrimento das atividades curativas e/ou assistenciais"'.

O Programa Médicos pelo Brasil ${ }^{7}$ institui a Agência para o Desenvolvimento da Atenção Primária à Saúde (Adaps), serviço social autônomo federal para prover médicos para a APS em locais de difícil lotação ou alta vulnerabilidade. Durante a formação, o profissional trabalhará por dois anos com bolsa de estudos, sem vínculo empregatício. O Conselho Deliberativo da Adaps terá representantes do Ministério da Saúde, dos Conselhos de Secretários de Saúde e do setor privado; ausentes, portanto, o Conselho Nacional de Saúde (CNS), usuários e trabalhadores.

Em comum a essas medidas e ao Programa Previne Brasil ${ }^{8}$, o caráter de "big bang" de contrarreformas em relação a políticas que ampliaram cobertura e acesso e buscaram promover mudança no modelo assistencial e maior controle do gasto público. O ambiente político na saúde é tenso e a disputa, acirrada. De um lado, flexibilidade e autonomia para a gestão local, defendidos pelo Ministério, Conselho Nacional de Secretarias Municipais de Saúde (Conasems) e entidades médicas; de outro, as críticas de sanitaristas, Conselhos de Secretários Municipais de Saúde do Rio de Janeiro e de São Paulo (Cosems/RJ e Cosems/SP) e do CNS, que apontam o caráter privatizante, mercadológico, seletivo e focalizado da APS proposta, na concepção de cobertura universal em saúde, em detrimento a de sistema de saúde universal almejado para o SUS ${ }^{5,10-13}$.

Este artigo analisa o Programa Previne Brasil $^{8}$, a partir das apresentações realizadas pela Secretaria de Atenção Primária à Saúde (SAPS) do Ministério da Saúde, da portaria que o instituiu e seus manuais instrutivos; e identifica contradições e alternativas para minimizar potenciais prejuízos às políticas vigentes. 


\section{Argumentos para implantação do Programa Previne Brasil}

A nova proposta de financiamento da APS foi divulgada em seminários realizados em vários estados, com a participação do Secretário de Atenção Primária, Erno Harzheim, de conselhos de secretários estaduais e municipais de saúde, gestores locais e representantes do movimento sanitarista.

Em defesa da medida, Harzheim baseou-se em duas críticas ao modelo vigente: i) Suposta "ineficiência" dos serviços em APS, considerando o grande número de usuários do SUS não cadastrados pelas equipes. Dos 50 milhões não cadastrados, 30 milhões seriam beneficiários de programas sociais, em situação de maior vulnerabilidade; ii) Baixa valorização do componente de desempenho das equipes, tendo em vista serem as transferências federais aos entes subnacionais majoritariamente calculadas com base em critérios populacionais.

Para Harzheim o cálculo atual de cobertura em APS, de 3.450 pessoas para cada eSF, seria um falseamento da realidade pois não lhes assegura a assistência; a exigência do cadastramento seria um estímulo ao cuidado real.

O Programa de Melhoria do Acesso e da Qualidade (PMAQ), que repassa recursos financeiros mediante alcance de metas, foi criticado pelo elevado número de indicadores e baixa capacidade de induzir melhorias. O modelo ora proposto romperia com essa lógica a partir do incremento, em valores nominais, de aproximadamente dois bilhões de reais ao orçamento para a APS em 2020, com novos critérios fundamentados em três eixos:

i) Substituição dos Pisos de Atenção Básica (PAB) Fixo e Variável pela Capitação Ponderada. Deixam de existir repasses regulares de base populacional e incentivos para custeio de eAB, Núcleo Ampliado de Saúde da Família (Nasf) e o PMAQ. Passa a ser considerado o cadastramento de cada cidadão na UBS correspondente. Há acréscimo do valor per capita e do fator de ponderação para menores de 5 anos ou maiores de 65 anos, beneficiários de programas sociais de transferência de renda, população de até dois salários mínimos vinculados ao Instituto Nacional de Seguridade Social (INSS), maior peso para municípios rurais.

ii) Incentivos do PMAQ serão substituídos por repasses condicionados ao desempenho em sete indicadores a serem instituídos em 2020, referentes a processos e resultados em Saúde da Gestante, da Mulher, da Criança e Doenças Crônicas. Até 2022, o número de indicadores chegaria a 21, com o monitoramento sobre Tuberculose, Infecções Sexualmente Transmissíveis e Saúde Bucal.

iii) Repasses vinculados a ações e programas de saúde contidos no PAB variável, passarão a compor o eixo de adesão a programas estratégicos, como os Programas Saúde na Hora; Apoio à Informatização e Qualificação dos Dados da APS - Informatiza APS e Programa de Formação em Residência Médica ou Multiprofissional em Odontologia e Enfermagem na Saúde da Família.

\section{Contradições e potenciais prejuízos ao financiamento da APS}

Ressalta-se o contexto de restrição orçamentária da EC 95 na relação com o prometido aporte adicional de 2 bilhões na Lei Orçamentária Anual (LOA) para a APS em 2020. O Instituto de Pesquisa Econômica Aplicada (IPEA) ${ }^{14}$ projetou redução dos gastos em ações e serviços públicos de saúde (ASPS) como percentual do Produto Interno Bruto (PIB) nos próximos 20 anos, projeção confirmada em dados apresentados por Ocké-Reis ${ }^{15}$.

A participação da União no financiamento do SUS, reduzida de 73\% para 43\% entre 1991 e 2017, foi acompanhada do aumento da alocação de recursos das esferas estadual (de 15\% para $26 \%$ ) e, principalmente, municipal (de 12\% para $31 \%$ ). Atualmente os estados têm aplicado percentuais próximos ao parâmetro mínimo de $12 \%$ da receita estadual (base de cálculo), e há um contencioso em relação a Minas Gerais e Rio de Janeiro; os municípios aplicaram recursos crescentes alcançando, em 2017, média em torno de $24 \%{ }^{16}$.

A capacidade de ampliação pelos municípios da aplicação de recursos próprios em APS está restringida também quando analisada em conjunto com as competências constitucionais de tributar e com a receita disponível de cada ente da federação, respectivamente: União (69\% e $57 \%$ ); estados (25\% e $25 \%$ ); e municípios $(6 \%$ e $18 \%$ ). Pela moda (variação mais frequente de uma distribuição), o financiamento da APS nos municípios se comporta da seguinte forma: $70 \%$ provêm do município e 30\%, da esfera federal ${ }^{16}$.

A SAPS prevê ganho para quase todos os municípios com o novo modelo, projetando-se um cenário de fronteira de eficiência irreal, com cadastramento de toda a população e alcance pleno das metas de indicadores que ainda não foram pactuados. Embora a população mais vulnerável seja tida como prioritária, não se mencionam 
indicadores como o Acompanhamento das Condicionalidades do Bolsa Família. Ademais, a proposta não explicita os custos de transação do Ministério e dos municípios, deixando de apontar os recursos necessários para a criação da Equipe de Apoio do Novo Financiamento no MS ou os gastos excedentes das secretarias municipais para ampliação de cadastros, muitas vezes com equipes terceirizadas por Organizações Sociais (OS).

Estudos $^{17,18}$ estimam perdas de $\mathrm{R} \$ 400$ milhões para municípios do estado do Rio de Janeiro e de R \$ 700 milhões para os de São Paulo, considerando a situação atual de cadastro em APS. Em cenário ótimo e improvável, com $100 \%$ de cadastro, restaria perda financeira em torno de 3\% no Rio de Janeiro e de $25 \%$ em São Paulo sobre os valores recebidos em 2018.

Necessário questionar o argumento do cadastramento na garantia do acompanhamento e cuidado aos usuários inscritos nas UBS. A parametrização automática de uma eSF como responsável por 2.000 a 4.000 pessoas pela nova proposta não significa acesso e atendimento efetivos, tampouco o cadastramento no modelo simplificado. A medida pode induzir a busca por cadastramento, sem incentivo financeiro federal significativo para aumentar o número de equipes e com a dificuldade real de seguimento do cuidado. A análise sobre a suposta ineficiência do cadastramento atual desconsidera os problemas gerenciais na manutenção de equipes completas, estrutura física de unidades e perdas de registros em transições de tecnologias como nas implantações do Sistema de Informações em Atenção Básica (Sisab) ou da Estratégia e-SUS.

A programação orçamentária federal para a APS em 2020 apresenta-se contraditória ao argumento da necessidade de maior valorização do desempenho. Em evento no Rio de Janeiro, Harzheim comparou os valores nominais do PMAQ em 2019 com os valores do eixo "desempenho" para 2020, com queda de $\mathrm{R} \$ 1.970,7$ para $\mathrm{R} \$$ $1.865,9$ bilhão. Apesar da programação de aumento nos anos seguintes, reitera-se que não há definição dos indicadores e suas metas.

$\mathrm{O}$ desestímulo à manutenção dos Nasf e a exclusão de fonoaudiólogos, fisioterapeutas e nutricionistas do Programa de Residência Multiprofissional elimina um diferencial positivo do sistema brasileiro e coloca em risco o princípio da integralidade, ademais com a previsão de vínculos precários por dois anos.

O ganho em flexibilidade e autonomia não se dará na organização da rede municipal pois há evidências de forte atuação do comando central na diretriz de capitação ponderada e intensiva normatização de programas como o Saúde na Hora, que encapsula as equipes em arranjos de distribuição de carga horária contrárias ao vínculo com as famílias, atributo essencial da APS.

Autores do campo do movimento sanitário apontam outras críticas: risco de "desfinanciamento"; impertinência em considerar o cadastro como via única de inserção ao SUS, desconsiderando seu caráter universal; provável direcionamento da prestação em APS para o setor privado através da conjugação entre cesta de serviços e lista de pacientes cadastrados; ausência de participação social e representação do $\mathrm{CNS}^{19}$; prejuízo ao planejamento e segurança de financiamento pelo fim do PAB fixo ${ }^{10,20}$. Afirma-se o teor inconstitucional ou no mínimo ilegal da medida, ao descumprir os preceitos da Lei Complementar 141/2012 e da Lei 8.142/1990, no que tange ao caráter deliberativo do CNS no controle das políticas de saúde, inclusive nos aspectos econômicos e financeiros.

\section{Considerações finais}

É improvável o recuo de um governo de perfil tecnocrata, onde as soluções técnicas obscurecem os problemas relacionados ao ajuste fiscal e à desigualdade de acesso, considerando o prazo de implantação do modelo em 2020.

Há necessidade de se criticar política e teoricamente a concepção dessa proposta no campo na economia da saúde e da saúde coletiva e de considerar o posicionamento de entidades do movimento da reforma sanitária eas iniciativas legislativas para revogação da Portaria $2.979 / 2019^{21}$. Propostas alternativas negociadas devem ressaltar que o cadastramento de usuários - contato inicial das eSF com a população adscrita - não pode condicionar o acesso ao SUS, tampouco servir ao repasse orçamentário regular.

O PAB fixo precisa ser mantido, de forma inegociável, pelo caráter estruturante do modelo pré-fixado. $\mathrm{Na}$ área hospitalar, a Portaria 3.410/2013 aponta o orçamento global como uma modalidade prevista, pela incerteza do pagamento por produção, ainda mais com os defasados valores da tabela do SUS. Em países com sistemas de saúde consistentes variam as modalidades com aproveitamento dos ônus e dos bônus de cada uma, mantida significativa parcela regular para assegurar a sustentabilidade do sistema ${ }^{22}$.

Por fim, sugere-se o direcionamento do incentivo financeiro ao desempenho para valorar o 
indicador de Acompanhamento das Condicionalidades do PBF, que além de promover o cadastramento da população mais vulnerável, garante o monitoramento de aspectos nutricionais de gestantes e crianças com medição de peso e crescimento, calendário vacinal e frequência escolar.

\section{Colaboradores}

MH De Seta, CO Ocke-Reis e ALP Ramos contribuíram igualmente na concepção, análise e redação do artigo, responsabilizando-se integralmente pelo seu conteúdo.

\section{Referências}

1. Hermida JF, Lira JS. Políticas Educacionais em Tempos de Golpe: Entrevista com Dermeval Saviani. Educ Soc 2018; 39(144):779-794.

2. Brasil. Ministério da Saúde (MS). Portaria no 2.436, de 21 de setembro de 2017. Aprova a Política Nacional de Atenção Básica, estabelecendo a revisão de diretrizes para a organização da Atenção Básica, no âmbito do Sistema Único de Saúde (SUS). Diário Oficial da União 2017; 22 set.

3. Brasil. Ministério da Saúde (MS). Portaria no 2.539 , de 26 de setembro de 2019. Altera as Portarias de Consolidação no 2e no 6, de 2017, para instituir equipe de Atenção Primária - eAP e dispor sobre o financiamento de equipe de Saúde Bucal - eSB com carga horária diferenciada. Diário Oficial da União 2019; 27 nov.

4. Brasil. Ministério da Saúde (MS). Portaria no 3.992, de 28 de dezembro de 2017. Altera a Portaria de Consolidação $n^{\circ}$ 6/GM/MS, de 28 de setembro de 2017, para dispor sobre o financiamento e a transferência dos recursos federais para as ações e os serviços públicos de saúde do Sistema Único de Saúde. Diário Oficial da União 2017; $28 \mathrm{dez}$.

5. Giovanella Ligia, Mendoza-Ruiz A, Pilar ACA, Rosa MC, Martins GB, Santos IS, Silva DB, Vieira JML, Castro VCG, Silva PO, Machado CV. Sistema universal de saúde e cobertura universal: desvendando pressupostos e estratégias. Cien Saude Colet 2018; 23(6):17631776.

6. Brasil. Ministério da Saúde (MS). Consulta pública sobre a Carteira de Serviços para a Atenção Primária à Saúde do Brasil [Internet]. [acessado 2019 nov 26]. Disponível em: http://189.28.128.100/dab/docs/portaldab/documentos/carteira_servico_da_APS_consulta_SAPS.pdf. 
7. Brasil. Medida Provisória $n^{\circ} 890$, de $1^{\text {o }}$ de agosto de 2019. Institui o Programa Médicos pelo Brasil, no âmbito da atenção primária à saúde, e autoriza o Poder Executivo federal a instituir serviço social autônomo denominado Agência para o Desenvolvimento da Atenção Primária à Saúde. Diário Oficial da União 2019; 01 ago.

8. Brasil. Ministério da Saúde (MS). Portaria no 2.979, de 12 de novembro de 2019. Institui o Programa Previne Brasil, que estabelece novo modelo de financiamento de custeio da Atenção Primária à Saúde no âmbito do SUS, alterando a Portaria de Consolidação no 6/ GM/MS, de 28 de setembro de 2017. Diário Oficial da União 2019; 13 nov.

9. Klein R. Big Bang Health Care Reform: Does It Work? The Case of Britain's 1991 National Health Service Reforms. Milbank Q1995; 73(3):299-337.

10. Lígia G, Bousquat A, Almeida PF, Melo EA, Medina MG, Aquino R, Mendonça MHM. Médicos pelo Brasil: caminho para a privatização da atenção primária à saúde no Sistema Único de Saúde? Cad Saude Publica 2019; 35(10):e00178619.

11. Morosini MVGC, Fonseca AF, Lima LD. Política Nacional de Atenção Básica 2017: retrocessos e riscos para o Sistema Único de Saúde. Saude Debate 2018; 42(116):11-24.

12. Mendes A, Carnut L, Guerra LDS. Reflexões acerca do financiamento federal da Atenção Básica no Sistema Único de Saúde. Saude Debate 2018; 42(n. esp. 1):224243.

13. Rede APS. Posicionamentos sobre a Carteira de Serviços da APS [Internet]. [acessado 2019 nov 26]. Disponível em: https://redeaps.org.br/2019/09/09/posicionamentos-sobre-a-carteira-de-servicos-da-aps/.

14. Vieira FS, Benevides RPS. Os impactos do Novo Regime Fiscal para o financiamento do Sistema Único de Saúde e para a efetivação do direito à saúde no Brasil. Brasília: Ipea; 2016. (Nota Técnica, nº 28).

15. Ocké-Reis CO. Piso constitucional da saúde caiu? [Internet]. Instituto de Medicina Social da UERJ; 2019 [acessado 2019 nov 26]. Disponível em: https://www. abrasco.org.br/site/wp-content/uploads/2019/11/ ocke_ipea-ims_01_11_19.pdf.

16. Ocké-Reis CO, Benevides RPS, Funcia FR. O piso constitucional da saúde caiu? Rio de Janeiro: Ipea; 2020. (Nota Técnica, no prelo).

17. Conselho de Secretarias Municipais de Saúde do Estado do Rio de Janeiro (Cosems/RJ). Análise da proposta de mudança na modalidade de transferência de recursos da APS. Documento para discussão [Internet]. Rio de Janeiro; 2019 [acessado 2019 nov 26]. Disponível em: http://www.cosemsrj.org.br/ assista-na-integra-do-seminario-proposta-do-ministerio-da-saude-da-mudanca-da-modalidade-detransferencia-de-recursos-financiamento-da-atencao -primaria-em-saude-que-debateu-o-tema/.
18. Conselho de Secretarias Municipais de Saúde do Estado de São Paulo (Cosems/SP). Nota Técnica COSEMS/SP: Novo modelo de financiamento da Atenção Primária em Saúde [Internet]. [acessado 2019 dez 10]. Disponível em: http://www.cosemssp.org.br/noticias/ nota-tecnica-cosems-sp-novo-modelo-de-financiamento-da-atencao-primaria-em-saude/.

19. Funcia FR. Novo modelo de financiamento da Atenção Primária à Saúde pactuado na Comissão Intergestores Tripartite (CIT) em 31 de outubro de 2019. Domingueira No 39 - Novembro 2019 [Internet]. [acessado 2019 nov 26]. Disponível em: http://idisa.org.br/domingueira/domingueira-n-39-novembro-2019?lang=pt.

20. Centro Brasileiro de Estudos de Saúde (Cebes). Carta ao Ministro da Saúde sobre os riscos da mudança de financiamento da Atenção Primária em Saúde [Internet]. [acessado 2019 nov 26]. Disponível em: http:// cebes.org.br/2019/10/carta-ao-ministro-da-saudesobre-os-riscos-da-mudanca-de-financiamento-da -atencao-primaria-em-saude/.

21. Padilha A. Projeto de Decreto Legislativo no 701, de 19 de novembro de 2019. Susta a Portaria $n^{\circ} 2.979 / G A-$ $B-M S$, de 12 de novembro de 2019, que estabelece novo modelo de financiamento de custeio da Atenção Primária à Saúde no âmbito do Sistema Único de Saúde, por meio da alteração da Portaria de Consolidação no 6/ GM/MS, de 28 de setembro de 2017 [Internet]. [acessado 2019 dez 12]. Disponível em: https://www.camara. leg.br/proposicoesWeb/prop_mostrarintegra;jsessionid $=0 A 131$ E69498FF816BFBA25E53C1434B0.proposicoesWebExterno2? codteor $=1834875 \&$ filename $=-$ Tramitacao-PDL+701/2019.

22. Uga MAD. Sistemas de alocação de recursos a prestadores de serviços de saúde - a experiência internacional. Cien Saude Colet 2012; 17(12):3437-3445.

Artigo apresentado em 16/01/2020

Aprovado em 15/02/2020

Versão final apresentada em 17/02/2020

Editores-chefes: Romeu Gomes, Antônio Augusto Moura da Silva 